\title{
Enhancing electricity network efficiency in sub- Saharan Africa through optimal integration of minigrids and the main grid
}

\author{
Madalitso Chikumbanje*, Damien Frame, Stuart Galloway \\ Department of Electronic and Electrical Engineering, University of Strathclyde, Glasgow, United Kingdom \\ *madalitso.chikumbanje@strath.ac.uk
}

\begin{abstract}
In the past two decades, grid extension and off-grid solutions, such as autonomous minigrids, have become main conduits for improving energy access in developing countries. Once universal energy access is mostly achieved in these countries, efficiency, reliability, quality of supply and flexibility will become increasingly prominent objectives in the planning and operation of their electricity networks. Some of these objectives, such as network efficiency, will be strongly influenced by the approach taken for the integration of the main grid and autonomous minigrids. This paper applies optimization techniques to investigate the impact of residual generation and load factor of an autonomous minigrid in the selection of an optimal point of grid connection to minimize energy losses.
\end{abstract}

Index Terms-minigrid, optimization, integration, distributed energy resources, energy access.

\section{INTRODUCTION}

In the past two decades, there has been significant progress in addressing the energy access gap, which exists in most developing countries [1]. For example, in 2017, there were 840 million people did not have access to electricity compared to 1.2 billion in 2010 [2]. The progress in energy access is due to a simultaneous application of both grid expansion and off-grid energy services such as minigrids and solar home systems (SHSs) [3]. The increasing role of off-grid systems in complementing the characteristically slow expansion of the main grid [4] is as a result of technical, business, policy and regulatory innovations in the recent past. Among other things, these include the reduction in prices for solar photovoltaic technology, increased application of Pay as You Go (PAYG) and other business models, remote monitoring and smart metering $[5,6]$. Any realistic chance of achieving universal access to electricity rests on the continued simultaneous deployment of both grid and off grid energy solutions [7, 8].

Beyond meeting electricity access goals through grid expansions and off-grid means, these two tracks will begin to converge [9]. For this reason, an investigation on "What will happen when these two converge?" becomes imperative [10, 11]. Recently, this question has attracted the attention of academics $[9,12]$, development agencies $[10,11]$ and thought leaders [13]. The main focus of these works has been policy and regulatory insight that would enable a smooth convergence of the grid and main grids. It is widely believed that smooth management of such convergence can lead to several benefits such as network efficiency, reliability and improved quality of supply [14]. Such benefits are essential for post electrification sub-Saharan Africa because they are of secondary importance right now as the main goal is to achieve universal access to electricity as soon as possible [15]. For example, Figure 1 shows that electricity network efficiency (measured as transmission and distribution losses) is a challenge in most developing countries like Nigeria (32\%), Malawi (23.5\%) and Ethiopia (20\%) compared to developed countries with mature networks like United Kingdom (7\%) and Ireland (8\%).

While addressing the policy and regulatory issues are fundamental, it is also essential to investigate how this convergence can deliver some of the associated benefits like network efficiency. In [14], they argue that the integration of minigrids and the main grid will lead to network efficiency because minigrids will introduce distributed energy resources (DERs) in the network that will serve loads locally. This argument ignores a well-established body of knowledge which states that depending on their size, location (relative to a known grid connection point), number and type, distributed energy resources can lead to both positive and negative impacts on a network [16]. Various authors have used this body of knowledge to optimize the benefits of DERs on established networks by varying the four parameters stated above $[17,18]$. However, all those parameters are already set in an autonomous minigrid before its integration with the main. The only exception being the point of connection between the minigrid the main grid. Achieving network efficiency gains during integration is, therefore, a choice between modifying the DERs which are already in the minigrid or selection of the point of grid infeed into the minigrid.

Between the two options mentioned above, selection of the point of grid connection is preferable because it reduces impact on any investments which will have already been made in the 


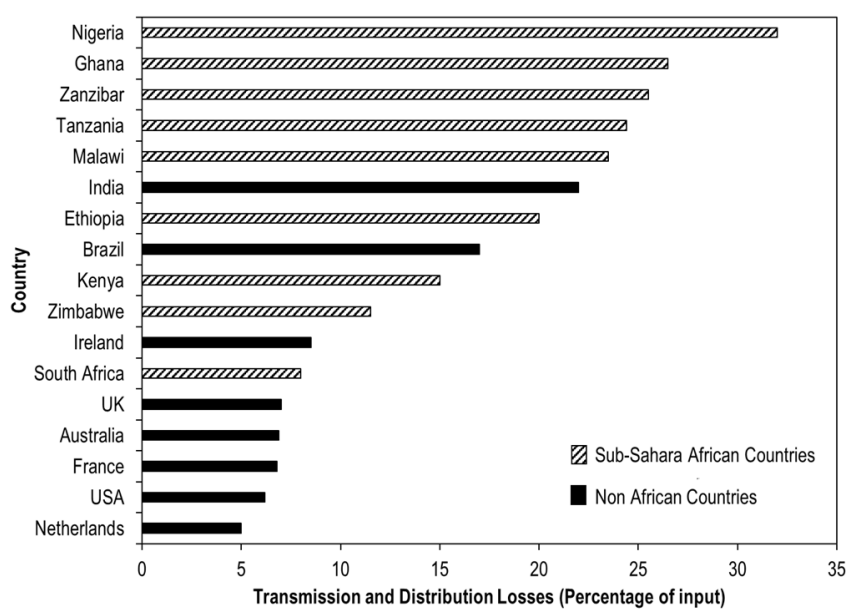

Figure 1. Comparison of electricity network losses [19]

minigrid. This paper uses optimization techniques to identify scenarios where selecting an optimal point of grid connection will lead to network efficiency in the integration of the main grid and minigrids in sub-Saharan Africa. The rest of the paper is organized as follows: Section II formulates the problem; Section III gives the solution methodology, Section IV introduces the cases studies; Section V presents the results and Section VI gives conclusions and future work.

\section{PROBlem Definition AND Formulation}

Consider a ten-node autonomous minigrid network shown in Figure $2 \boldsymbol{G}$, is the local power source connected to node 1 . Under islanded operations, the total power losses $\left(P_{L}\right)$ in the network is given by (1), where $n$ is the individual branch of the network with resistance $r_{n}, V_{n}$ is the receiving end voltage of the branch, $P_{n}$ and $Q_{n}$ represent the active and reactive power flowing through each branch, and $N$ is the total number of branches.

Upon the arrival of the main grid, any of the available ten nodes can become a point of grid infeed into the local network as long as it has the necessary number of phases. If the main grid connects to the same node as the incumbent generation (node 1 in this case) power losses in the network will still be given by (1) as the incoming grid will not affect any power flows in the local network. This is because the reference bus for the power flow calculation remains the same for both islanded and grid connected operation.

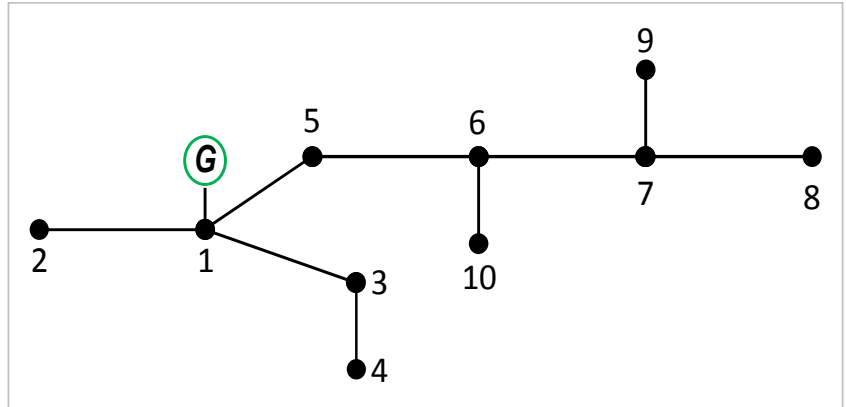

Figure 2. A ten-node minigrid network
When the grid is connected to a different node, apart from the one with local generation, power will flow differently in the network. In turn, this will also affect power losses. Notably, these changes will be in the branches making the path between the point of grid connection and local generation source. The network branches will then split into two sets. The first set, consisting of branches whose power flow is influenced by the output of the local generator, denoted by $\psi$, and the second set whose power flow is not affected by the production of the local generation. In this case, the power losses in the network will be given by (2) where $P_{g}$ and $Q_{g}$ are the active and reactive power output of the local generator.

$$
\begin{gathered}
P_{L}=\sum_{n=1}^{N} \frac{r_{n}}{V_{n}^{2}}\left(P_{n}^{2}+Q_{n}^{2}\right) \\
P_{L}=\sum_{n \notin \psi} \frac{r_{n}}{V_{n}^{2}}\left(P_{n}^{2}+Q_{n}^{2}\right)+\sum_{n \in \psi} \frac{r_{n}}{V_{n}^{2}}\left(\left(P_{n}-P_{g}\right)^{2}+\left(Q_{n}-Q_{g}\right)^{2}\right)
\end{gathered}
$$

To identify an optimal point of grid connection, energy losses over 24 hours are minimized, as follows, where $h$ represents the hour of the day.

$$
\min f=\sum_{h=1}^{24} P_{L}^{h}
$$

s.t.

$$
\begin{gathered}
0.95 \leq V_{n} \leq 1.05 \\
I_{n} \leq I_{\max } \\
P_{g}^{\text {min }} \leq P_{g} \leq P_{g}^{\text {max }}
\end{gathered}
$$

Energy losses are preferred to power losses as they take into account the time variation of loads as well as some generation sources such as solar photovoltaic which is the renewable technology of choice in sub-Saharan minigrid. The objective function is subject to constraints (4) - (6), where (4) ensures that the voltage in the network is within a window of $+/-5 \%$ of the nominal voltage. On the otherhand, (5) and (6) ensure that thermal limits of all equipment in the network are respected at all times. In (5) $I_{n}$ is the current through a network branch, and $I_{\text {max }}$ is the maximum acceptable current through the branch.

\section{Solution Methodology}

Power system optimization problems can be solved using either mathematical techniques such as calculus methods, linear and non-linear programming methods, dynamic programing method and integer programming method; or heuristic techniques such as Genetic Algorithm (GA), Simulated Annealing(SA) and Particle Swarm Optimization (PSO) [20]. Each of these techniques has its advantages and disadvantages which are well documented in the literature [21].

The minimization problem in (3) - (6) is non-linear because the evaluation of losses, currents and voltages in a power network involves solving power flow equations which are nonlinear themselves. As well, the main grid can only be connected to a finite number of three-phase nodes in the local network, making the elements in the solution space discrete. Therefore, 
finding the optimal point of grid connection considering energy losses will involve solving a non-linear optimization problem with a discrete solution space.

For a smaller minigrid network, this problem can be solved by direct enumeration. However, this becomes less practical for a relatively sizeable minigrid network. Related non-linear problems such as network reconfiguration [22] and distributed generation placement [18] are solved using heuristic methods like GA. Despite their inherent weaknesses, such as lack of any indication on the optimality gap, GA is ideal for problems with discrete variables and the possibility of several local critical points. This advantage has seen GA being applied in the distribution system phase balancing module of a commercial power system analysis software in [23]. In this paper, a GA is used to solve the problem defined and formulated in Section II.

\section{CASE STUdies AND SCEnARIOS}

The methodology presented in Section III was applied on two networks that were modified to represent a trunk and branch topology of a minigrid. Each network model was simulated with three different load profiles, to observe the impact of load factor in the selection of the point of grid connection. Since the source of power in a minigrid can be a hybrid of renewable and non-renewable resources, the integration of the main grid will lead to the decommissioning of the non-renewable component for environmental reasons [10]. As such, eleven different levels of residual generation penetration with respect to the peak demand have also been evaluated. The justification of the selected case study networks and scenarios is as follows:

\section{A. Networks}

So far, scholars and practitioners are yet to agree on a single minigrid definition [24]. Consequently, there is no standard minigrid network which can be used to study their integration with the grid [25]. According to [26], minigrids can have capacities of anything from $10 \mathrm{~kW}$ to $10 \mathrm{MW}$. Therefore, the two networks used in this paper attempt to capture networks on both ends of the spectrum. The IEEE 33 bus network, in Figure 3 has a peak demand of $3715 \mathrm{~kW}$, representing a relatively higher capacity minigrid. The Low Voltage (LV) network (adapted from [25]), in Figure 4 has its loads adjusted to a peak of $250 \mathrm{~kW}$ represents low capacity minigrids. Both networks were modified by introducing a generation source on Node 1 which are primarily slack buses for their normal application. This modification allows for a trunk and branch topology which is one of the minigrid topologies in developing countries [4].

\section{B. Minigrid Residual Generation}

In [10] not all of the generation capacity available during autonomous operation of a minigrid will make it into grid connected operation. Some capacity (e.g. diesel generators) are likely to be decommissioned for environmental reasons while others due to technical incompatibility with the incoming grid [27]. In this paper, the possible reduction in residual generation capacity is accounted for by assuming scenarios of 0 to $100 \%$ (with increments of $10 \%$ ) penetration of residual installed generation with respect to peak demand. As well, the paper mainly focuses on residual generation capacity of solar photovoltaic with a deterministic bell-like profile.

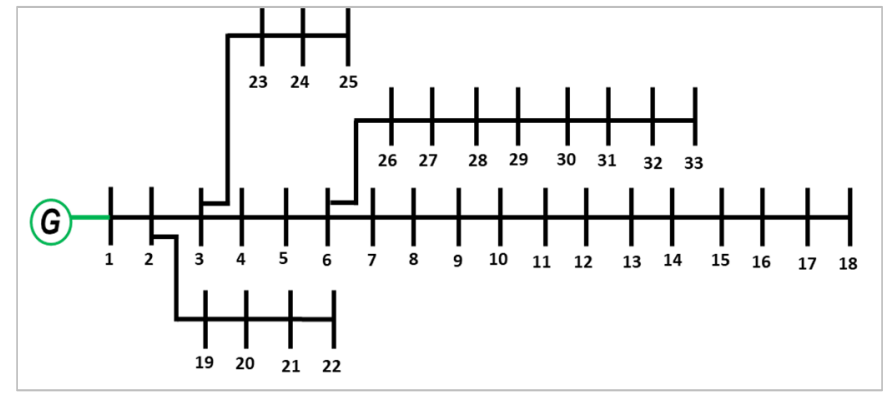

Figure 3. IEEE 33 bus test network

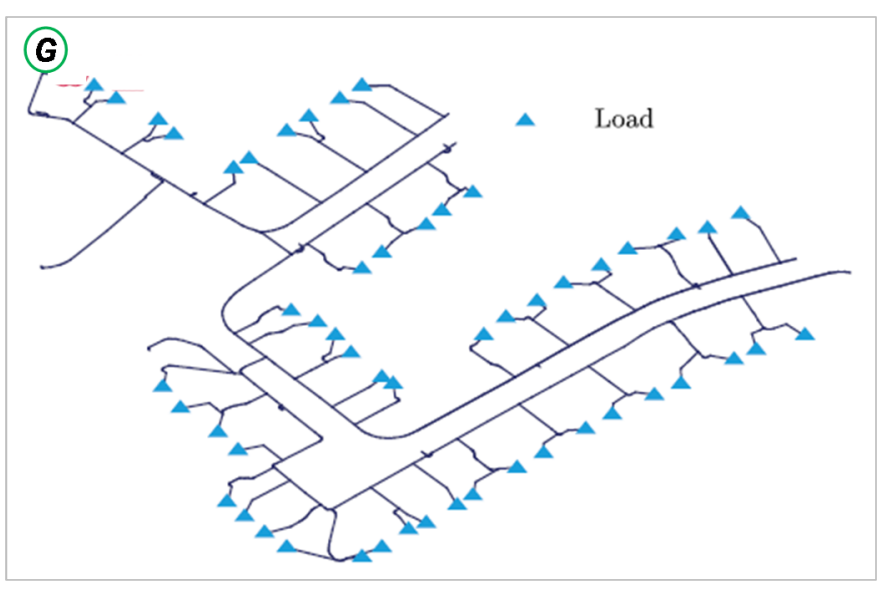

Figure 4. IEEE Low Voltage Test Network (Graphic adapted from [28])

\section{Minigrid Load Factor}

Besides the magnitude of the demand, this paper captures the impact of the daily behaviour of load on the choice of the point of grid connection. This is important because [26] suggests that Load Factor (LF) for minigrids has potential to grow from the existing $22 \%$ to $80 \%$. In this study, three load profiles with LFs of $21 \%, 46 \%$ and $69 \%$ [29] were used.

\section{SIMULATION RESUlTS AND DisCUSSION}

The results from this study are presented and discussed from two perspectives. The first perspective concerns the identified optimal point of grid connection and the second perspective concerns the actual network efficiency gains that each identified point of connection can bring to the minigrid.

\section{A. Optimal Points of Connection}

The optimal points of connection for the different scenarios of load factors and residual minigrid generation are presented in TABLE 1. These results demonstrate that both networks have a specific node which is ideal for the integration of the main grid in most of the scenarios. Specifically, these are node 6 for the 33 bus network and node 280 for the LV network. In the few cases where the popular node is not selected, the optimal node is found to be on the path between the popular node and the point to which the local generation is located. For example, node 3 is between nodes 1 and 6 in the 33 bus network, and node 101 is on the path between nodes 1 and 280. Therefore, every minigrid with the topologies presented in this paper will have a specific node to which the grid can be connected and guarantee network efficiency for most of the possible scenarios. 
TABLE 1. OPTIMAL POINTS OF CONNECTION FOR DIFFERENT SCENARIOS

\begin{tabular}{|c|c|c|c|c|c|c|}
\hline \multirow{3}{*}{ 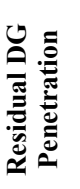 } & \multicolumn{6}{|c|}{ Optimal Point of Grid Connection } \\
\hline & \multicolumn{3}{|c|}{$\begin{array}{c}33 \text { Bus Network } \\
\text { (Fig. 3) }\end{array}$} & \multicolumn{3}{|c|}{$\begin{array}{c}\text { LV Network } \\
\text { (Fig. 4) }\end{array}$} \\
\hline & $\begin{array}{c}\text { Low } \\
L F\end{array}$ & $\begin{array}{l}\text { Med. } \\
\text { LF }\end{array}$ & $\begin{array}{c}\text { High } \\
\text { LF }\end{array}$ & $\begin{array}{l}\text { Low } \\
\text { LF }\end{array}$ & $\begin{array}{c}\text { Med. } \\
\text { LF }\end{array}$ & $\begin{array}{c}\text { High } \\
\text { LF }\end{array}$ \\
\hline 0 & 6 & 6 & 6 & 280 & 280 & 280 \\
\hline 10 & 6 & 6 & 6 & 280 & 280 & 280 \\
\hline 20 & 6 & 6 & 6 & 280 & 280 & 280 \\
\hline 30 & 6 & 6 & 6 & 280 & 280 & 280 \\
\hline 40 & 4 & 6 & 6 & 280 & 280 & 280 \\
\hline 50 & 3 & 6 & 6 & 114 & 280 & 280 \\
\hline 60 & 2 & 6 & 6 & 101 & 280 & 280 \\
\hline 70 & 1 & 6 & 6 & 32 & 280 & 280 \\
\hline 80 & 1 & 6 & 6 & 32 & 280 & 280 \\
\hline 90 & 1 & 3 & 6 & 32 & 280 & 280 \\
\hline 100 & 1 & 3 & 6 & 32 & 114 & 280 \\
\hline
\end{tabular}

TABLE 1 also shows that the most prevalent node is not considered optimal for a combination of low and medium load factors and a residual photovoltaic penetration of more than $30 \%$ and $90 \%$ respectively. Such scenarios will leave the ones with evaluating the point of grid connection with a decision to make. Whether to connect the grid to the suggested point of connection or the prevalent node for the grid. The former will be following the optimization methodology while the latter will be supported by the most likely assumption that the coming of the grid will lead to an increase in demand and load factor which will then reduce the penetration level of the residual generation and lead to network efficiency.

\section{B. Network Efficiency Gains}

To assess the network efficiency gains, energy losses from the optimized points of connection were compared with energy loss if the grid was connected to node 1 in both networks (where the generator is connected). These results are presented in Fig. 5, 6 and 7 where Network 1 represents the IEEE 33 bus network and Network 2 is the LV network.

The network results presented Figures 5, 6 and 7 show that optimal grid location has more efficiency gains for a minigrid with high load factor. In all scenarios, the efficiency gains tend to diminish with an increasing level of residual generation penetration. The results also show that optimized losses are below or equal to the base cases except for the LV network low load factor case. This can be attributed to the network having some voltage violations at the set peak loading. These violations may be expected as at the time of integration with the grid, minigrids networks will have a different look from the ones that were initially deployed due to load growth and network expansion to accommodate new connections.

In the specific case presented in this paper, the solver attempts to find a solution that respects the given constraints of voltage and thermal limits, hence optimized energy losses are higher than base losses. However, across all scenarios, the reported results clearly show that such eventualities will be an exception rather than the rule as load factors in minigrids are expected to increase with a growing interest in productive use of energy and electric cooking.
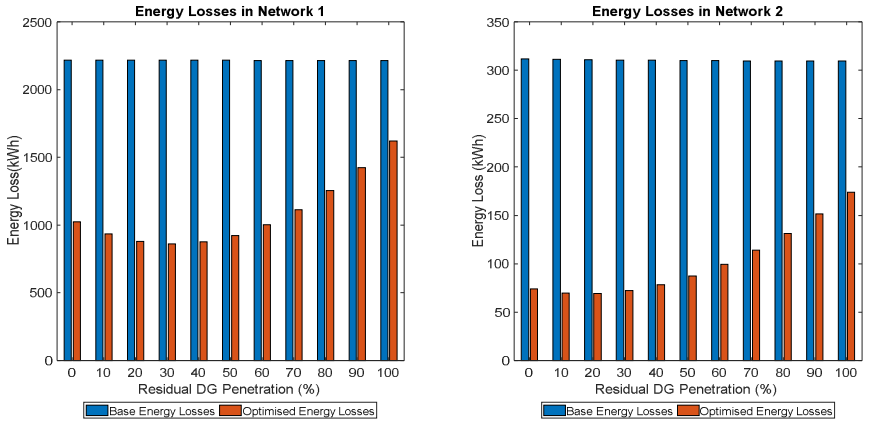

Figure 5. Base and optimized energy losses for high LF
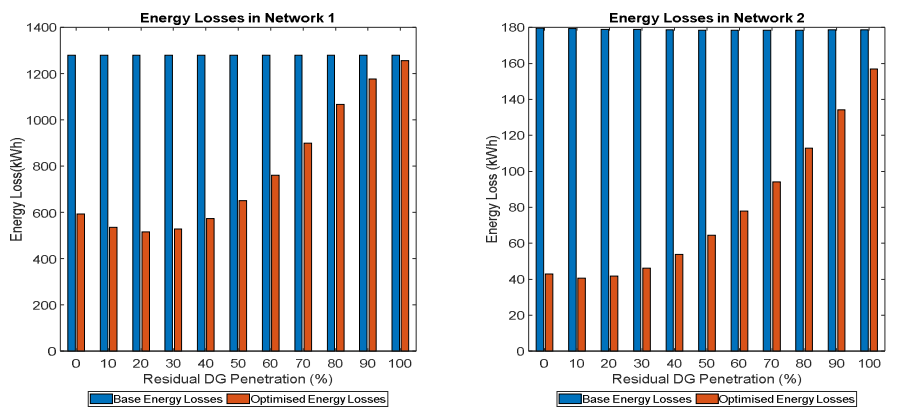

Figure 6. Base and optimized energy losses for medium LF
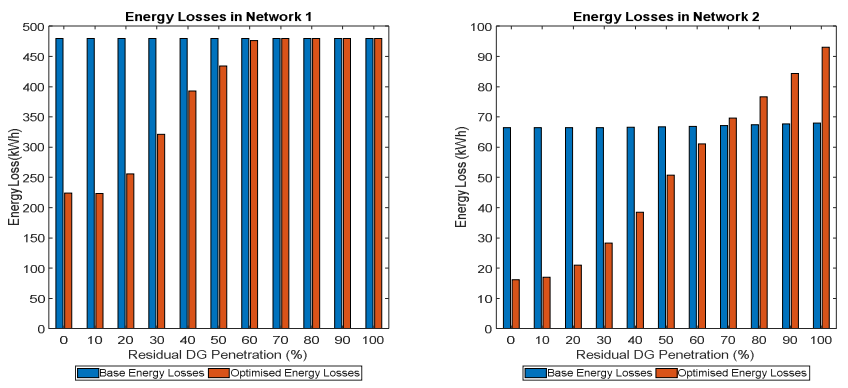

Figure 7. Base and optimized energy losses for low LF

\section{CONCLUSIONS AND FUTURE WORK}

Using case studies and scenarios, this paper has shown that selection of an optimal point of integrating a minigrid with the main grid has got potential to enhance the future network efficiency of incumbent minigrids in developing countries. For most of the scenarios investigated, it can be concluded that every network (with similar topologies as investigated in this study) will have a 'prevalent node' that can ensure loss reduction upon integration with the main grid. The only scenarios that give a different result are those with low load factors and high residual photovoltaic penetration. In these cases, it will be even more important for the decision maker to consider future load growth within the minigrid network in addition to the optimization results. Although not reported in this study, the results indicate that connecting the main grid to the prevalent node of the minigrid would lead to long term network efficiency benefits as the load grows and residual photovoltaic generation penetration reduces.

In addition to the insights presented in this paper, there are other issues that remain untested. For example, only one 


\section{IEEE PES/IAS PowerAfrica}

Enhancing electricity network efficiency in sub-Saharan Africa through optimal integration of minigrids and the main grid

generation technology (solar photovoltaic) without energy storage has been considered. But, minigrids often contain a range of different generation technologies, such as wind and mini-hydro, and most are likely to also contain storage or diesel generators to smooth the effects of intermittent generation. Furthermore, this paper only considers energy losses as an objective for selecting the point of grid connection. Other objectives such as reliability of supply to the minigrid customers, and voltage deviation and imbalance are not considered. Future work will look to understand the impact of different generation technologies and multiple objectives on the selection of the optimal point of minigrid connection to the main grid in sub-Saharan Africa.

\section{ACKNOWLEDGMENT}

The authors would like to thank the Scottish Funding Council and the University of Strathclyde for providing funding to conduct this research.

\section{REFERENCES}

[1] International Energy Agency, "Energy Access Outlook 2017: From Poverty to Prosperity," in "World Energy Outlook Special Report," International Energy Agency (IEA), Paris2017, Available: https://www.iea.org/publications/freepublications/publication/WE O2017SpecialReport EnergyAccessOutlook.pdf.

[2] IEA, IRENA, UNSD, The World Bank, and WHO, "Tracking SDG7: the energy progress report 2019," Washington DC2019, Available: 05/TrackingSDG7 report-2019.pdf.

[3] S. Bhattacharyya and D. Palit, "The nexus of grid, mini-grids and off-grid options for expanding electricity access," 2019.

[4] H. Louie, Off-Grid Electrical Systems in Developing Countries. Springer, 2018.

[5] International Finance Corporation (IFC), "From Gap to Opportunities: Business Models for Scaling up Energy Access," The World Bank2012.

[6] P. Hollberg, "Swarm grids - Innovation in rural electrification," Independent thesis Advanced level (degree of Master (Two Years)) Student thesis, 2015.

[7] J. Peters, M. Sievert, and M. A. Toman, "Rural electrification through mini-grids: Challenges ahead," Energy Policy, vol. 132, pp. 27-31, 2019/09/01/2019.

[8] J. Rozenberg and M. Fay, "Beyond the Gap : How Countries Can Afford the Infrastructure They Need while Protecting the Planet " 2019.

[9] J. Tjäder, S. Ackeby, and C. Bastholm, "The role and interaction of microgrids and centralized grids in developing modern power systems," presented at the India Smart Grid Week 2016, 15-19 March 2016 at Manekshaw Centre, DhaulaKuan, New Delhi, India, 2016, $2016 . \quad$ Available: http://urn.kb.se/resolve?urn=urn:nbn:se:du-23497

[10] B. Tenenbaum, C. Greacen, and D. Vaghela, "Mini-Grids and Arrival of the Main Grid : Lessons from Cambodia, Sri Lanka, and Indonesia," The World Bank, Washington, DC.2018, Available: https://openknowledge.worldbank.org/handle/10986/29018

[11] Rockefeller Foundation, "Beyond Off-grid: Integrating Mini-grids with India's Evolving Electricity System," 2017, Available: https://www.rockefellerfoundation.org/report/integrating-minigrids-indias-evolving-electricity-system/.

[12] T. Levin and V. M. Thomas, "Can developing countries leapfrog the centralized electrification paradigm?," (in English), Energy for Sustainable Development, vol. 31, pp. 97-107, Apr 2016.

[13] O. Zinaman et al., "Power Systems of the Future," The Electricity Journal, vol. 28, no. 2, pp. 113-126, 2015.

[14] Vivid Economics and ARUP, "Opportunities to enhance electricity network efficiency," London2015.

[15] E. Day, "Moving beyond energy access - the challenge and impact of unreliable electricity in emerging economies," 2020.

[16] M. Castilla and A. C. Z. d. Souza, Microgrids design and implementation, Cham, Switzerland: Springer, 2019. [Online]. Available: SpringerLink. Restricted to UC campuses https://doi.org/10.1007/978-3-319-98687-6.

R. Viral and D. K. Khatod, "Optimal planning of distributed generation systems in distribution system: A review," Renewable and Sustainable Energy Reviews, vol. 16, no. 7, pp. 5146-5165, 2012/09/01/2012.

[18] P. S. Georgilakis and N. D. Hatziargyriou, "Optimal Distributed Generation Placement in Power Distribution Networks: Models, Methods, and Future Research," Ieee Transactions on Power Systems, vol. 28, no. 3, pp. 3420-3428, Aug 2013.

[19] Millenium Challenge Account - Malawi (MCA-M), "ESCOM FINOP Project - Loss Study," MCA-M, Lilongwe2015.

[20] H. Seifi and M. S. Sepasian, Electric Power System Planning (Power Systems). Springer, 2011.

[21] M. Fathi and H. Bevrani, Optimization in Electrical Engineering. Springer, 2019.

[22] M. Abdelaziz, "Distribution network reconfiguration using a genetic algorithm with varying population size," Electric Power Systems Research, vol. 142, pp. 9-11, 2017.

[23] DIgSILENT, PowerFactory 2017 User Manual. Gomaringen: DIgSILENT GmbH, 2016.

[24] S. C. Bhattacharyya, "Mini-Grids for the Base of the Pyramid Market: A Critical Review," (in English), Energies, vol. 11, no. 4, Apr 2018.

[25] K. Schneider et al., "Analytic considerations and design basis for the IEEE distribution test feeders," IEEE Transactions on power systems, vol. 33, no. 3, pp. 3181-3188, 2017.

[26] Energy Sector Management Assistance Programme., "Mini Grids for Half a Billion People : Market Outlook and Handbook for Decision Makers," World Bank, Washington, DCESMAP Technical Report;014/19, 2019, Available: https://openknowledge.worldbank.org/handle/10986/31926

[27] C. Greacen, R. Engel, and T. Quetchenbach, "A Guidebook on Grid Interconnection and Islanded Operation of Mini-Grid Power Systems Up to 200kW," Lawrence Berkeley National Laboratory, California2013, Available: https://publications.lbl.gov/islandora/object/ir\%3A158819/datastr eam/PDF/view.

[28] M. Nijhuis, M. Gibescu, and S. Cobben, "Gaussian mixture based probabilistic load flow for LV-network planning," IEEE Transactions on Power Systems, vol. 32, no. 4, pp. 2878-2886, 2016.

[29] N. J. Williams, P. Jaramillo, B. Cornell, I. Lyons-Galante, and E. Wynn, "Load Characteristics of East African Microgrids," (in English), 2017 Ieee Pes Powerafrica Conference, pp. 236-241, 2017. 